\title{
Pediatric advanced stage nasopharyngeal carcinoma - case report
}

\author{
${ }^{1}$ Department of Pediatrics, Clinical \\ Hospital Centre Rijeka, Croatia \\ ${ }^{2}$ National Cancer Institute, Aviano, Italy \\ ${ }^{*}$ Corresponding author: \\ jelena.roganovic1@ri.t-com.hr \\ Tel.: + 38551659214 \\ Fax.: + 38551623126
}

Jelena Roganovići ${ }^{*}$, Nuša Matijašić1 ${ }^{1}$, Mascarin Maurizio ${ }^{2}$

Received: 23 September 2015

Accepted: 17 November 2015

Key words: Nasopharyngeal carcinoma • Rare tumors • Child.

\begin{abstract}
Objective. Nasopharyngeal carcinoma is an extremely rare pediatric malignancy predominantly occurring in adolescent males. Its multifactorial pathogenesis is most strongly associated with the exposure to Epstein-Barr virus in genetically susceptible hosts. In younger patients, more aggressive biological behavior has been observed, although the overall survival is better compared to adults. Due to its rarity and nonspecific clinical presentation, the diagnosis in children is often delayed and misinterpreted. Case report. We report a case of a 16-year-old boy with stage IVB nasopharyngeal carcinoma. He presented with a painless palpable neck mass, nasal congestion and a history of occasional epistaxis and headaches. Four years after the completion of a multimodal treatment, the patient is in complete remission. Conclusion. Although exceedingly rare, pediatricians should consider nasopharyngeal carcinoma in the differential diagnosis of palpable neck masses, especially in male adolescents. A multidisciplinary approach in the diagnosis, treatment, supportive care and follow-up is of utmost importance.
\end{abstract}

\section{Introduction}

Nasopharyngeal carcinoma (NPC) is a rare pediatric malignancy accounting for less than $1 \%$ of all pediatric neoplasms (1). The age-adjusted annual incidence rate increases with age, reaching its peak in adolescents (2). Its multifactorial etiology comprises genetic predisposition, epigenetic alternations related to Epstein-Barr virus (EBV) and exposure to environmental factors. Compared to adults, pediatric NPC is biologically more aggressive and generally diagnosed in advanced stages $(3,4)$. The current treatment protocols have resulted in excellent outcome (2). We report a diagnostic and therapeutic approach to a male adolescent with advanced stage NPC.

\section{Case report}

A 16-year-old boy was admitted to the Department of Head and Neck Surgery, Clinical Hospital Centre Rijeka, due to a one-month painless swelling on the right side of the neck. The mass showed no signs of regression following two-week antibiotic treatment. Additionally, for the previous three months the patient had been complaining of nasal congestion and obstruction, as well as occasional epistaxis and headaches, attributed to a seasonal allergy. His personal and family medical history were unremarkable.

Ultrasound showed bilateral cervical lymphadenopathy. Lymph node fine needle aspiration demonstrated carcinoma cells. The patient underwent node biopsy. His- 
topathological and immunohistochemical examination set the diagnosis of undifferentiated NPC, World Health Organization (WHO) type III (Figure $1 \mathrm{~A}-\mathrm{D}$ ).

The boy was transferred to the Division of Hematology and Oncology, Department of Pediatrics, Rijeka, for further examination and treatment. Upon admission, he was in a good general condition, with an audible inspiratory stridor. Bilateral firm painless cervical lymph node conglomerates were palpable (left 10x6 cm, right $4 \times 4 \mathrm{~cm}$ in size). Complete blood count, kidney and liver function tests, lactate dehydrogenase, ferritin and copper were within reference values, as well as the tumor markers (carcinoembryonic antigen, neuron-specific enolase, alpha-fetoprotein and beta human chori- onic gonadotropin). Hypergammaglobulinemia was present with immunoglobulin (Ig) G level of $18.1 \mathrm{~g} / \mathrm{l}$ (normal range 7-16 $\mathrm{g} / \mathrm{l})$. Serological testing was positive for IgG and IgA viral capsid antigen (VCA) and IgG anti-EBV nuclear antigen (EBNA); IgM anti-VCA, IgM anti-EBNA and IgG anti-early antigen (EA) were negative. No circulating plasma EBV DNA was detected. Polymerase chain reaction (PCR) identified EBV DNA in the tumor tissue.

The multislice computed tomography (MSCT) of the head and neck confirmed bilateral cervical lymphadenopathy, and revealed a nasopharyngeal tumor 37.6 x 31.7 x $25.2 \mathrm{~mm}$ in size (Figure 2). Detailed imaging studies (chest and spine X-ray, abdominal ultrasound, CT of the chest and the abdomen,

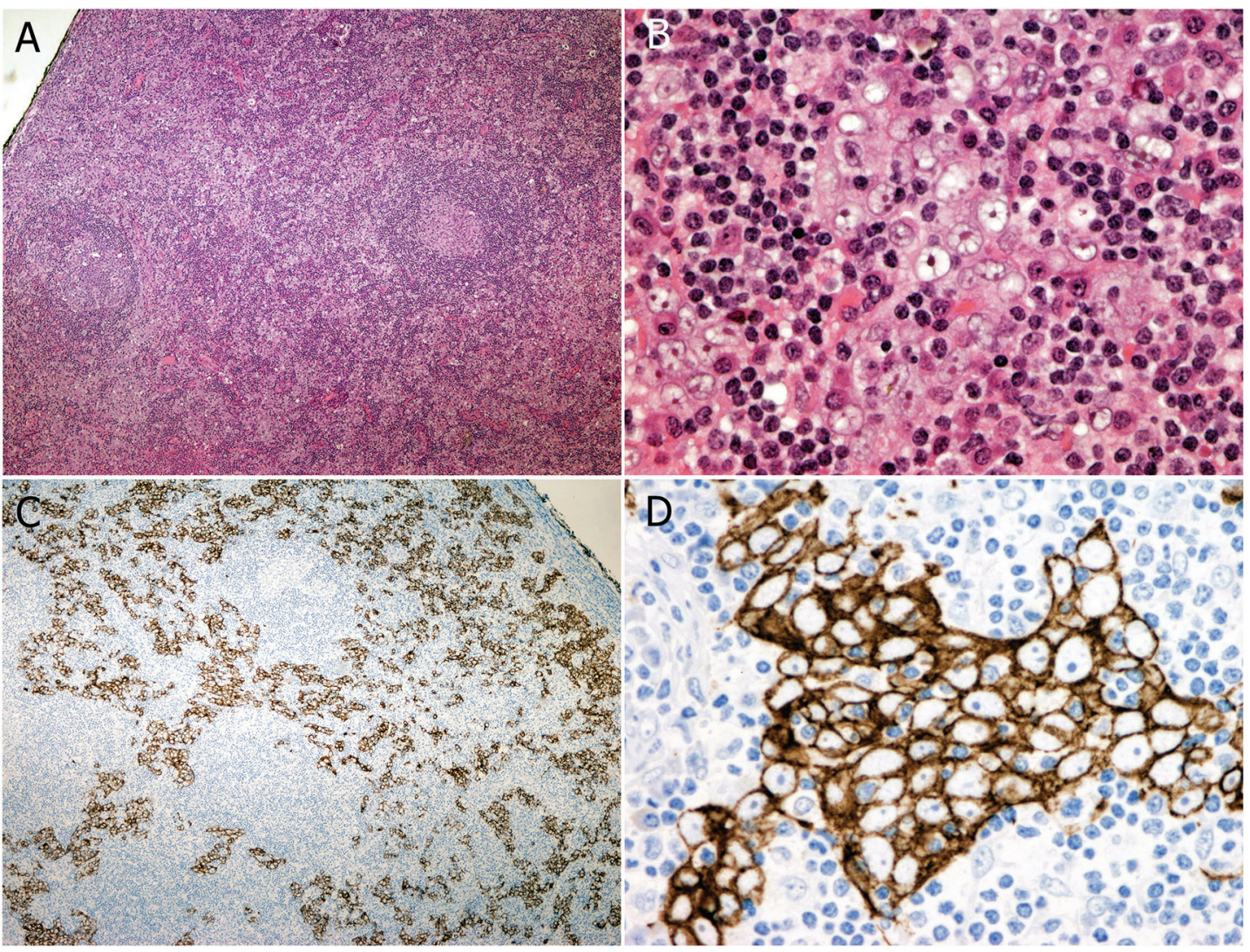

Figure 1 Metastasis of nasopharyngeal carcinoma to the lymph node. (A) Islands of tumor cells infiltrate the sinuses and lymph node parenchyma. Tumor cells exhibit large, vesicular nuclei with prominent red nucleolus and a moderate amount of pale eosinophilic cytoplasm (B, magnification $x 400)$. Immunohistochemically, tumors cells show strong cytoplasmic positivity with epithelial markers for keratin /AE1/AE3) (C, magnification $\mathrm{x} 40$; D, magnification $\mathrm{x} 400$ ). 
bone scintigraphy) excluded the presence of distant metastases. Bone marrow aspiration was negative for malignant infiltration. A revision of the histopathological findings confirmed the diagnosis of undifferentiated NPC. According to the TNM classification, the tumor was classified as T2 (parapharyngeal extension), N3 (lymph node metastases larger than $6 \mathrm{~cm}$ ) and M0 (no distant metastases), stratifying the patient into IVB stage and the high-risk group.

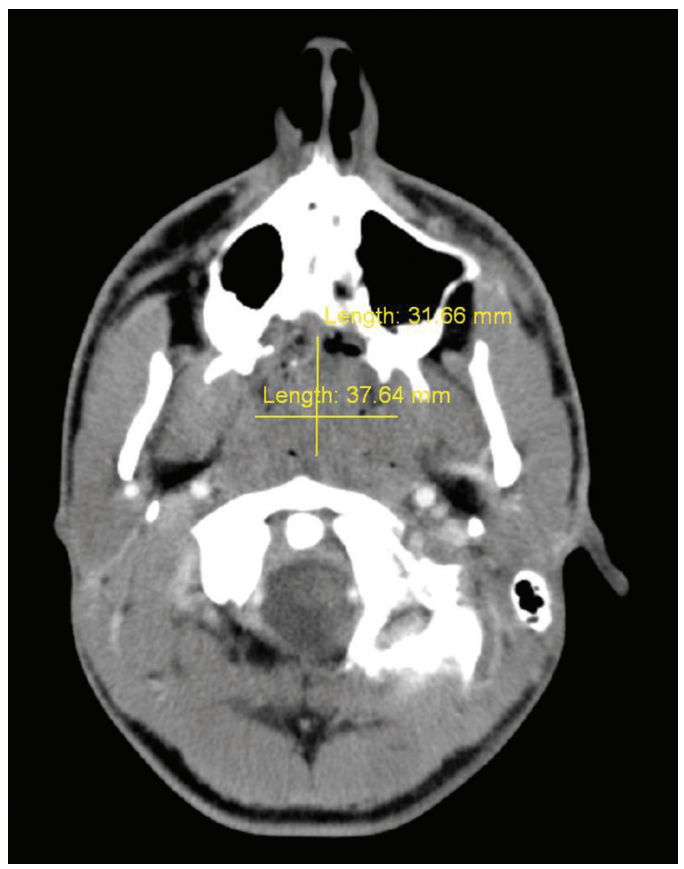

Figure 2 Multislice computed tomography (MSCT) of the head showing a nasopharyngeal tumor measuring $37.6 \times 31.7 \mathrm{~mm}$.

The boy was treated according to NPC2003-GPOH protocol. He received neoadjuvant chemotherapy consisting of three courses of cisplatin $\left(100 \mathrm{mg} / \mathrm{m}^{2}\right.$, day 1$)$, 5-fluorouracil (1.000 mg/m²/day, day 1-5), and folinic acid. During the treatment the patient developed deep right brachial vein thrombosis, which was treated with low molecular weight heparin for 3 months. He had several episodes of febrile neutropenia, and steroid diabetes during the neoadjuvant treatment.
Follow-up MSCT demonstrated a partial response. Photon external radiotherapy - tomotherapy was performed at the National Cancer Institute Aviano, Italy. A cumulative dose of 7000 cGy was delivered to the nasopharynx and left neck by conventional fractionation (33 fractions, 5 fractions/week), with concomitant cisplatin $\left(20 \mathrm{mg} / \mathrm{m}^{2}\right.$, day 1-3) during the first week and the last week of irradiation. After irradiation, the patient received recombinant interferon (IFN) beta for 6 months. CT performed 6 weeks after the end of radio-chemotherapy and at the end of IFN treatment showed no evidence of a tumor. Four years after the completion of the treatment, the patient is in complete remission. He takes replacement therapy for hypothyroidism.

\section{Discussion}

NPC is an extremely rare pediatric neoplasm that arises from the epithelial cells of the nasopharynx. The age adjusted annual incidence rate increases with age, being 0.1 per million at age 0 to 9 years, 0.8 per million at age 10 to 14 years, and 1 to 2 per million at age 15 to 19 years (2). The median age at diagnosis is 15.3 years (1).

The incidence of NPC is characterized by gender, racial, ethnical and geographic variations, demonstrating its multifactorial pathogenesis. Males are two to three times more frequently affected than females (3). NPC is more common in black children (4). It is endemic in a few well-defined regions of China, Southeast Asia and Arctic region, while intermediate incidence rates are seen in North Africa and parts of the Mediterranean basin $(3,5)$. Studies conducted in these regions have found a positive association between consumption of salt-preserved fish containing significant levels of nitrosamines and NPC risk $(3,6)$. In addition, a constant link has been described between the frequency of human leukocyte antigen 
(HLA) class I genes in certain populations and the risk of developing NPC (7). The strongest association has been established between NPC and EBV infection; circulating free EBV DNA is detected in more than $90 \%$ of advanced stage NPC patients. Titer levels of antibodies to EBV IgA-VCA and IgA-EA have been widely used as diagnostic and prognostic markers. Quantification of EBV DNA using real-time PCR is highly sensitive and specific for NPC, and is used in clinical management of NPC patients. High EBV DNA load at diagnosis or detectable viral load post-treatment is associated with poor survival and frequent relapse (8-10).

Pediatric NPC is different from its adult counterpart. It is biologically more aggressive and usually presents in an advanced stage. Approximately $87 \%$ of pediatric NPC is an undifferentiated subtype, which is more commonly associated with elevated EBV titer, confirming a prior viral infection in genetically susceptible children $(4,11)$. Furthermore, children are at higher risk of developing therapy-related complications, including secondary cancers. Nevertheless, pediatric patients have a significantly greater overall and relapse-free survival compared to adults $(4,12)$. The most commonly described symptoms in children are: neck mass, headache, tinnitus, hearing loss, nasal obstruction, cranial nerve palsy, diplopia and facial anesthesia. Due to the nonspecific symptoms, the median time to diagnosis is 4.8 months (13).

The treatment of pediatric NPC has undergone drastic changes in the last few decades. Until the early 1990's, patients were mostly treated by irradiation, but then chemotherapy was introduced. NPC-2003$\mathrm{GPOH}$ is the current treatment protocol applied in most European countries. It includes neoadjuvant chemotherapy, radiochemotherapy and IFN beta. The results are superior to the outcomes of published results from other pediatric NPC study groups, with an event-free survival rate of
$92.4 \%$ and overall survival rate of $97.1 \%$ at a median follow-up of 30 months (14). The combined therapy is well tolerated. Acute side effects are mainly leucopenia, mucositis and nausea, while chronic side effects are hearing loss and hypothyroidism $(2,15)$. In order to reduce toxicity, children have recently been treated by intensity-modulated radiotherapy (IMRT), which offers better volume coverage of the tumor, while protecting nearby healthy tissue (16). In addition, lower doses are administered without locoregional failures (16).

This paper presents an advanced stage, high-risk pediatric NPC. The disease initially presented as a bilateral cervical lymphadenopathy. Histopathological and immunohistochemical examination established the diagnosis of undifferentiated NPC. PCR identified EBV DNA in the tumor tissue, dictating the addition of EBV-directed immunotherapy with IFN to standard therapy. Four years after the end of the treatment the patient is in complete remission with acceptable toxicity, i.e. hypothyroidism resulting from radiotherapy. Our case indicates that NPC, although very rare, should be considered in children with solid neck masses. EBV serology as well as imaging methods are required to set up a treatment plan. Currently, the preferred imaging modality for disease staging is magnetic resonance imaging (MRI). Positron emission tomography-computed tomography (PET-CT) may underestimate the extent of the tumor and regional lymphadenopathy compared to MRI at the time of diagnosis, but is sensitive and specific for follow-up (17). Despite the low rate of relapses/recurrences, long-term follow-up is recommended, as well as endocrine monitoring, due to iatrogenic hypothyroidism.

\section{Conclusion}

NPC is a very rare pediatric malignancy characterized by its biological aggressive- 
ness. The current treatment protocols have significantly improved the prognosis for young patients. Further prospective multiinstitutional studies are required in order to standardize treatment approaches in children with different stages and histological subtypes of NPC, as well as to investigate the later toxicity of the treatment.

Acknowledgement: We are grateful to dr Nives Jonjić, School of Medicine, Rijeka, for performing histopathological and immunohistochemical diagnosis, and providing histopathological images and their descriptions.

Authors' contributions: Conception and design: JR, $\mathrm{NM}$; Acquisition, analysis and interpretation of data: JR, NM; Drafting the article: NM; Revising it critically for important intellectual content: JR, MM.

Conflict of interest: The authors declare that they have no conflict of interest.

\section{References}

1. Guruprasad B, Tanvir P, Rohan B, Kavitha S, Naik SM, Appaji L. Paediatric nasopharyngeal carcinoma: an 8-year study from a tertiary care cancer centre in South India. Indian J Otolaryngol Head Neck Surg. 2013;65(Suppl 1):131-4.

2. Brennan B. Nasopharyngeal carcinoma. Orphanet J Rare Dis. 2006;1:23.

3. Chang ET, Adami HO. The enigmatic epidemiology of nasopharyngeal carcinoma. Cancer Epidemiol Biomarkers Prev. 2006;15(10):1765-77.

4. Sultan I, Casanova M, Ferrari A, Rihani R, Rodriguez-Galindo C. Differential features of nasopharyngeal carcinoma in children and adults: a SEER study. Pediatr Blood Cancer. 2010;55(2):279-84.

5. Adham M, Kurniawan AN, Muhtadi AI, Roezin A, Hermani B, Gondhowiardjo S, et al. Nasopharyngeal carcinoma in Indonesia: epidemiology, incidence, signs, and symptoms at presentation. Chin J Cancer. 2012;31(4):185-96.

6. Jia WH, Luo XY, Feng BJ, Ruan HL, Bei JX, Liu WS, et al. Traditional Cantonese diet and nasopharyngeal carcinoma risk: a large-scale casecontrol study in Guangdong, China. BMC Cancer. 2010;10:446.

7. Li X, Fasano R, Wang E, Yao KT, Marincola FM. HLA associations with nasopharyngeal carcinoma. Curr Mol Med. 2009;9(6):751-65.
8. Leung SF, Chan KC, Ma BB, Hui EP, Mo F, Chow $\mathrm{KC}$, et al. Plasma Epstein-Barr viral DNA load at midpoint of radiotherapy course predicts outcome in advanced-stage nasopharyngeal carcinoma. Ann Oncol. 2014;25(6):1204-8.

9. Hutajulu SH, Kurnianda J, Tan IB, Middeldorp JM. Therapeutic implications of Epstein-Barr virus infection for the treatment of nasopharyngeal carcinoma. Ther Clin Risk Manag. 2014;10:72136.

10. Yip TT, Ngan RK, Fong AH, Law SC. Application of circulating plasma/serum EBV DNA in the clinical management of nasopharyngeal carcinoma. Oral Oncol. 2014;50(6):527-38.

11. Bray F, Haugen M, Moger TA, Tretli S, Aalen OO, Grotmol T. Age-incidence curves of nasopharyngeal carcinoma worldwide: bimodality in low-risk populations and aetiologic implications. Cancer Epidemiol Biomarkers Prev. 2008;17(9):2356-65.

12. Downing NL, Wolden S, Wong P, Petrik DW, Hara W, Le QT. Comparison of treatment results between adult and juvenile nasopharyngeal carcinoma. Int J Radiat Oncol Biol Phys. 2009;75(4):106470 .

13. Liu W, Tang Y, Gao L, Huang X, Luo J, Zhang S, et al. Nasopharyngeal carcinoma in children and adolescents - a single institution experience of 158 patients. Radiat Oncol. 2014;9:274.

14. Buehrlen M, Zwaan CM, Granzen B, Lassay L, Deutz P, Vorwerk P, et al. Multimodal treatment, including interferon beta, of nasopharyngeal carcinoma in children and young adults: preliminary results from the prospective, multicenter study NPC-2003-GPOH/DCOG. Cancer. 2012;118(19):4892-900.

15. Peng G, Wang T, Yang KY, Zhang S, Zhang T, Li Q, et al. A prospective, randomized study comparing outcomes and toxicities of intensity-modulated radiotherapy vs. conventional two-dimensional radiotherapy for the treatment of nasopharyngeal carcinoma. Radiother Oncol. 2012;104(3):286-93.

16. Casanova M, Bisogno G, Gandola L, Cecchetto G, Di Cataldo A, Basso E, et al. Rare Tumors in Pediatric Age Group. A prospective protocol for nasopharyngeal carcinoma in children and adolescents: the Italian Rare Tumors in Pediatric Age (TREP) project. Cancer. 2012;118(10):2718-25.

17. Cheuk DK, Sabin ND, Hossain M, Wozniak A, Naik M, Rodriguez-Galindo C, et al. PET/CT for staging and follow-up of pediatric nasopharyngeal carcinoma. Eur J Nucl Med Mol Imaging. 2012;39(7):1097-106. 